\title{
Tuberous sclerosis complex misdiagnosed as multiple metastases in a cervical cancer patient: case report and literature review
}

\author{
Ting Ye ${ }^{1 \#}$, Liwen Feng ${ }^{1 \#}$, Miao Peng ${ }^{1}$, Siyue Yuan ${ }^{1}$, Yuting Chen ${ }^{1}, \mathrm{Xi} \mathrm{Liu}^{2}$, Jing Chen ${ }^{1}$ \\ ${ }^{1}$ Cancer Center, Union Hospital, Tongji Medical College, Huazhong University of Science and Technology, Wuhan, China; ${ }^{2}$ Department of \\ Radiology, Union Hospital, Tongji Medical College, Huazhong University of Science and Technology, Wuhan, China \\ "These authors contributed equally to this work. \\ Correspondence to: Xi Liu. Department of Radiology, Union Hospital, Tongji Medical College, Huazhong University of Science and Technology, \\ Wuhan 430022, China. Email: liuxi027@163.com; Jing Chen. Cancer Center, Union Hospital, Tongji Medical College, Huazhong University of \\ Science and Technology, Wuhan 430022, China. Email: chenjingwh@hust.edu.cn.
}

\begin{abstract}
Tuberous sclerosis complex (TSC) is an autosomal dominant disorder that affects multiple organs and is caused by inactive mutations in the TSC1 or TSC2 genes. The main symptoms of TSC are neurocutaneous syndrome and benign hamartoma formation. Notably, malignancy is not an indication of TSC. In this article, we present the case of a 48-year-old female with cervical cancer (CC) combined with TSC, who was misdiagnosed with multiple metastases. Toe masses, pelvic nodules, and multiple osteogenic lesions were initially observed. Multi-site puncture biopsies and a toe amputation were performed; the pathology results did not indicate malignancy. Subsequently, hypomelanotic macules on the back, subependymal nodules (SENs), ungual fibromas, multiple renal cysts, and sclerotic-bone-lesions (SBLs) of the skull, and vertebrae were observed, leading to a diagnosis of TSC. Given that TSC is a benign disease and has not yet caused any organ disfunction, no special treatment was provided to this patient. After a follow-up period of almost 65 months, the patient's quality of life remained good without therapy. Oncologists should pay attention to benign diseases in the face of multiple lesions to reduce misdiagnosis and overtreatment. In addition, TSC may interact with CC through molecular mechanisms, such as the mammalian target of rapamycin (mTOR) pathway.
\end{abstract}

Keywords: Hyperplastic lesions; multiple nodules; tuberous sclerosis complex (TSC); case report

Submitted Aug 06, 2021. Accepted for publication Oct 16, 2021.

doi: 10.21037/apm-21-2814

View this article at: https://dx.doi.org/10.21037/apm-21-2814

\section{Introduction}

Tuberous sclerosis complex (TSC) is a rare disease affecting multiple systems. It is mainly characterized by neurocutaneous syndrome and benign hamartoma formation. Despite limited novel data on its epidemiology, TSC affects almost 2 million people worldwide and has a birth incidence of 1 in 6,000-10,000 (1,2). TSC is caused by a loss-of-function mutation in the TSC1 or TSC2 genes that encode hamartin and tuberin, respectively (3). As it may potentially involve any organ, each clinical manifestation of TSC is distinctive and individual. In reality, many cases will not be diagnosed due to the mild clinical symptoms and obscurity of the disease. The diagnostic criteria for TSC were updated at the 2012 International TSC Consensus Conference, which agreed that identifying of a pathogenic mutation in TSC1 or TSC2 is sufficient to make a definitive diagnosis (3). Note that mutations are not detected in $10-25 \%$ of individuals with clinical TSC, which does not exclude TSC. The clinical diagnostic criteria included 11 major features and 6 minor features. A definitive diagnosis is made if a patient has 2 major features or 1 major feature

\footnotetext{
$\wedge$ ORCID: 0000-0002-7279-7050.
} 


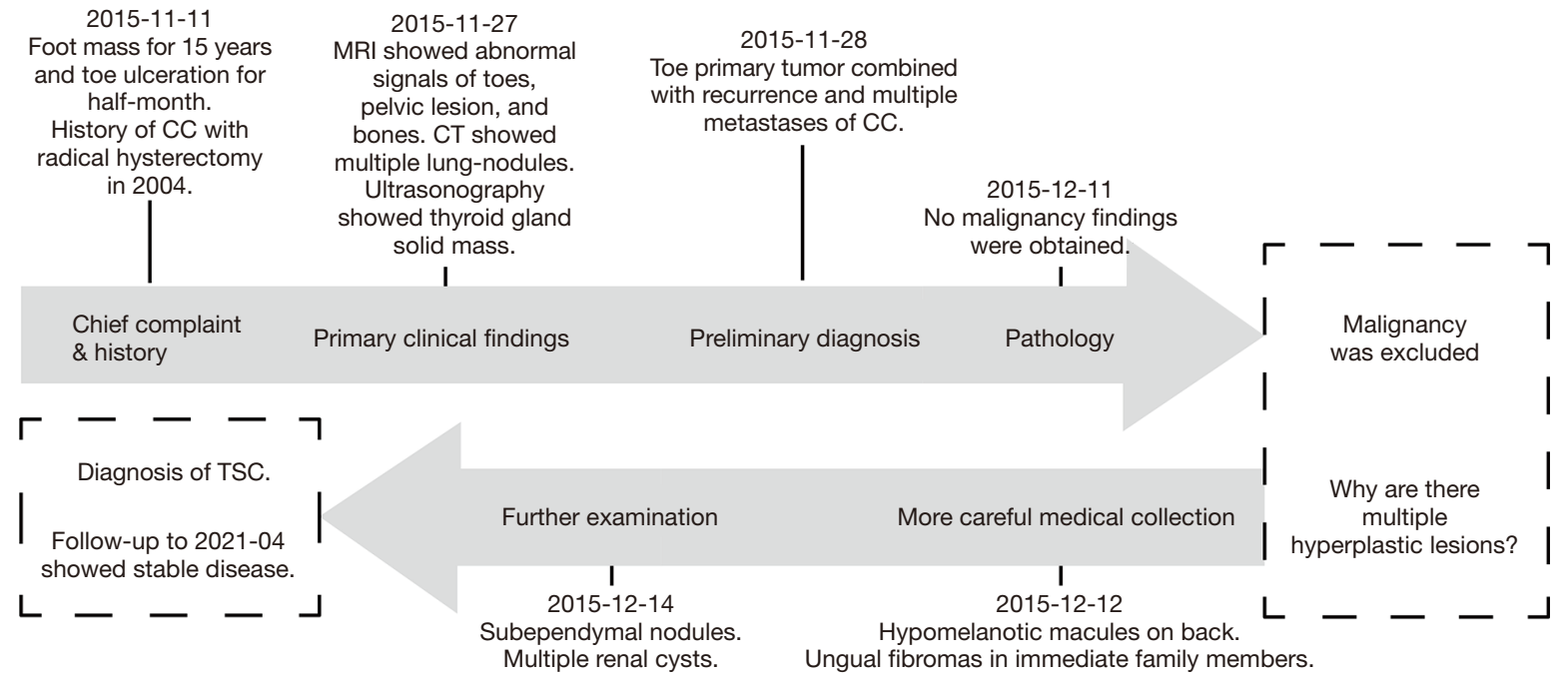

Figure 1 Timeline of the clinical course. CC, cervical cancer; TSC, tuberous sclerosis complex; MRI, magnetic resonance imaging; CT, computed tomography.

with $\geq 2$ minor features; a possible diagnosis is made if the patient has either 1 major feature or $\geq 2$ minor features. The major features included: hypomelanotic macules $(\geq 3$, at least $5 \mathrm{~mm}$ diameter), angiofibromas ( $\geq 3$ ) or fibrous cephalic plaque, ungual fibromas $(\geq 2)$, shagreen patches, multiple retinal hamartomas, cortical dysplasias, subependymal nodules (SENs), subependymal giant cell astrocytoma (SEGA), cardiac rhabdomyoma, lymphangioleiomyomatosis (LAM), angiomyolipomas $(\geq 2)$. The minor features included: "confetti" skin lesions, dental enamel pits $(\geq 3)$, intraoral fibromas $(\geq 2)$, retinal achromic patches, multiple renal cysts, nonrenal hamartomas.

In this article, we present the case of a patient with cervical cancer (CC) combined with TSC, who underwent a radical hysterectomy, and was misdiagnosed with a soft tissue tumor with CC recurrence and metastasis based on imaging and clinical examinations. After careful observation following the patient's diagnosis, TSC was confirmed, and metastasis was excluded. Ultimately, the patient was free of tumors and had a good quality of life in the 65-month follow-up period. There were similar reports about the TSC misdiagnosed. Olubunmi reported a case of TSC in a 13-year-old girl that was misdiagnosed as neurofibromatosis because of her skin lesions (4); however, this report mainly showed neurocutaneous syndrome, and the differential diagnosis of benign tumors was not described. Herein, we present a case of TSC, who was misdiagnosed with multiple metastases. Oncologists should pay attention to the differential diagnosis of benign diseases if multiple lesions are observed, especially when the clinical symptoms are atypical to reduce misdiagnosis and overtreatment and avoid causing psychological trauma to patients. In addition, some advances are discussed in relation to imaging performance, molecular mechanisms, and the potential association between TSC and CC. We present the following article in accordance with the CARE reporting checklist (available at https://dx.doi.org/10.21037/apm-21-2814).

\section{Case presentation}

In November 2015, a 48-year-old female patient was admitted with a foot mass of 15 years and toe ulceration of half a month. Figure 1 shows a timeline of the patient's clinical course. The patient reported that she had a history of CC and had undergone a radical hysterectomy in 2004. Her general condition was otherwise good, and she had no other symptoms. On physical examination, cauliflower-like masses on the great toes of both feet were observed, and the left great toe was ulcerated and foul-smelling (see Figure 2). The imaging results of the patient are shown in Figure 3. Contrast-enhanced magnetic resonance imaging (MRI) on November 27, 2015 showed an abnormal soft tissue signal of $19 \times 26 \mathrm{~mm}$ and $8 \times 5 \mathrm{~mm}$ in the left and right great toes, respectively, and an enhanced scan showed heterogeneous enhancement. In addition, the patient had soft tissue masses of $45 \times 28 \mathrm{~mm}$ in front of the sacrum, and bone destruction of the lumbar (see Figure $3 A, 3 B$ ), sacrum, and pelvis was observed. A computed tomography (CT) scan revealed 
multiple solid nodules that were suspected to be metastases in both lungs (see Figure 3C), and an ultrasonography showed a $50 \times 30.6 \mathrm{~mm}$ solid mass with calcification in the left lobe of the thyroid gland.

Based on the imaging results and the patient's history of CC, pelvic lymph node recurrence and multiple bone metastases were suspected. The preliminary diagnosis was as follows: (I) toe masses; (II) multiple bone destruction: possible bone metastasis; (III) multiple pulmonary nodules: possible pulmonary metastasis; (IV) abnormal pelvic

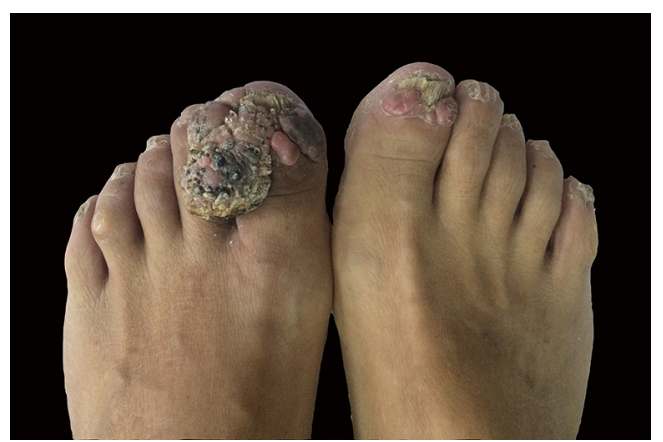

Figure 2 Masses on both feet at admission in November 2015. lymph nodes: possible recurrence of CC; and (V) thyroid mass. In view of the long course of the toe masses and the recent apparent rapid growth, soft tissue tumors with local primary and recent malignant transformation were also considered. A diagnosis of toe primary tumors combined with the recurrence and multiple metastases of CC was first considered. To further examine the masses, biopsies of the right toe, sacrum, and pelvic mass were performed on November 19, 2015; however, no evidence of malignancy was found. Due to the limited puncture tissue and the fact that the left great toe could not be preserved due to the severe ulceration, the patient elected to undergo an amputation of the left great toe on December 1, 2015 (see Figure 4 for the pathological picture). Malignancy of the foot, the recurrence of CC and bone metastases were subsequently excluded. On December 11, 2015, a puncture biopsy of the thyroid was performed, and the pathology results indicated a nodular goiter.

To determine why the patient presented with multiple systemic nodules that were pathologically suggestive of hyperplastic lesions, a more careful physical examination was undertaken, and the patient's medical history was reviewed. Consequently, hypomelanotic macules were
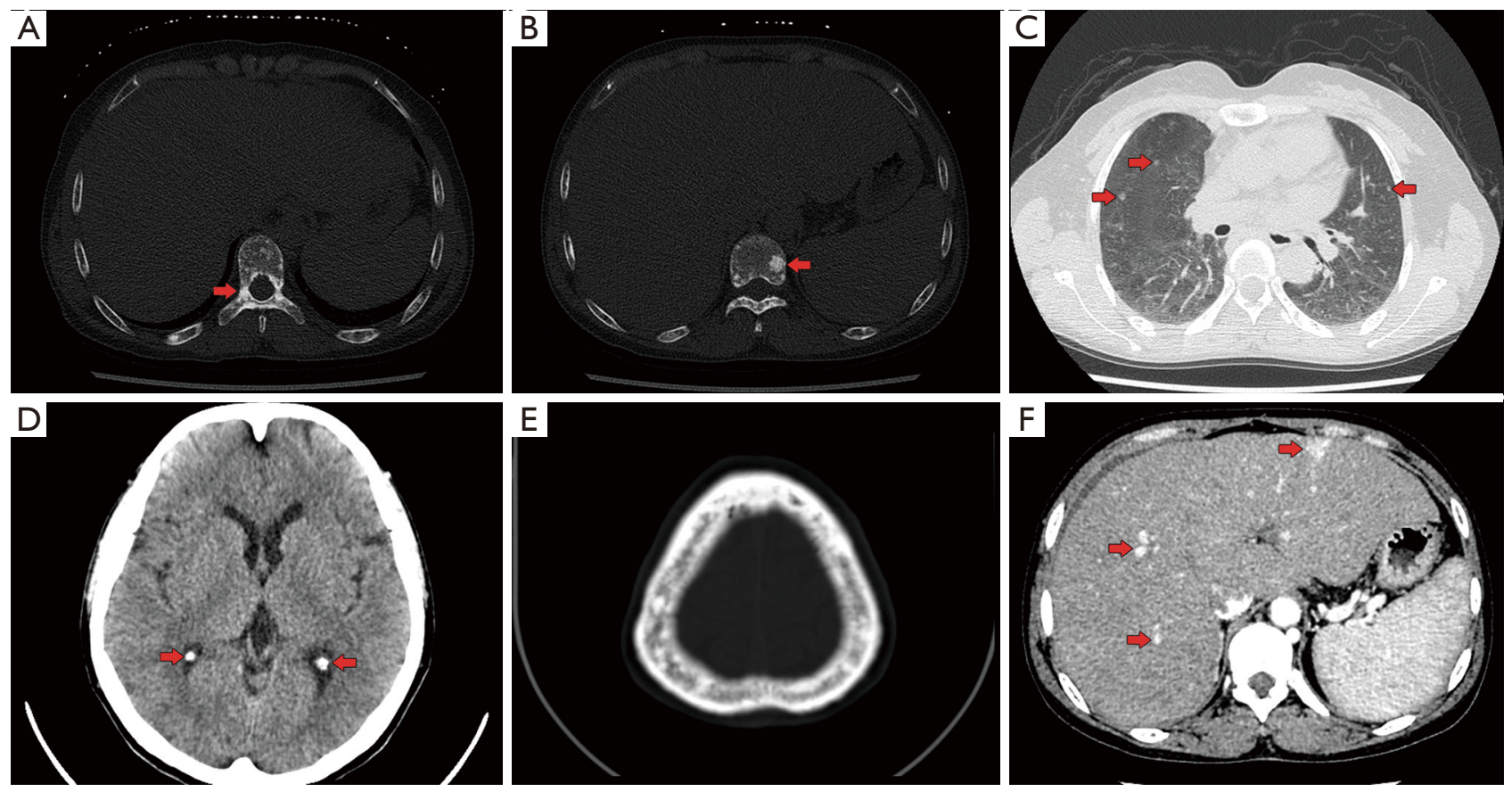

Figure 3 CT scans showed multiple nodules. (A,B) Multiple bone destruction on the lumbar; (C) multiple solid nodules in both lungs; (D) SENs; (E) multiple bone destruction on the skull; (F) multiple heterogeneous nodules with heterogeneous enhancement. The red arrows point to the lesions. CT, computed tomography; SENs, subependymal nodules. 


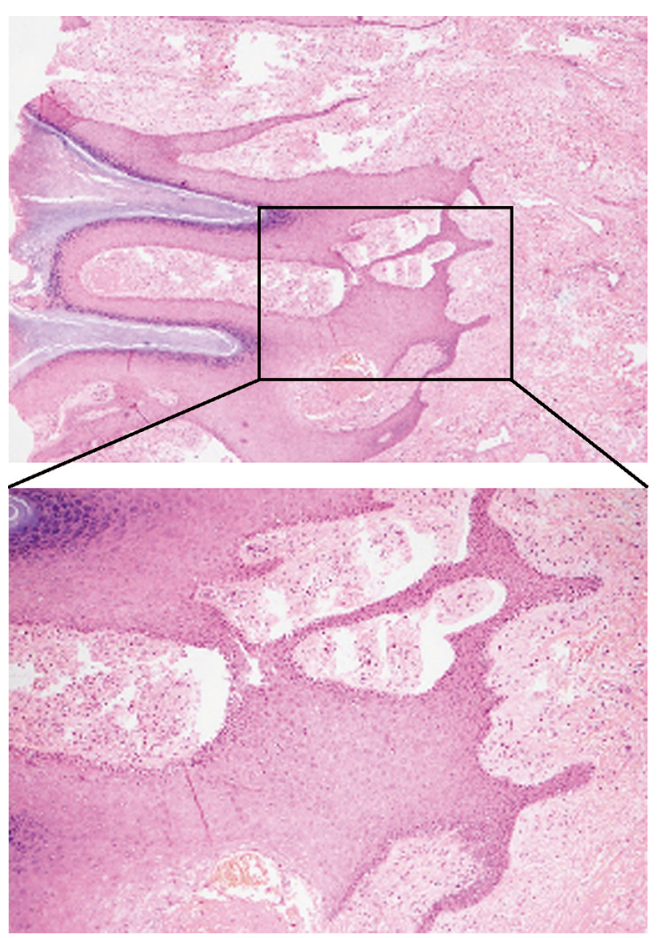

Figure 4 Pathological results for the left great toe. Optical microscopy findings, $\mathrm{H} \& \mathrm{E}$ staining. It is covered in squamous papillomatous hyperplasia. In some significant areas, thickening of the cuticle was also observed (original magnification 200x), and vascular and fibrous hyperplasia was observed in the dermis (original magnification 400x). H\&E, hematoxylin and eosin.

observed on the patient's back, and ungual fibromas were observed in her immediate family members. These characteristics all suggested the possibility of TSC. Thus, we further examined the patient's brain, heart, kidney, and eyes. CT scans showed calcification at the margin of the left ventricle (SENs) (see Figure 3D). An ultrasound showed a normal heart but multiple renal cysts. The results of a funduscopy were generally normal. The patient was eventually diagnosed with TSC. Unfortunately, genetic testing of TSC1 and TSC2 was not performed because our patient had reached a clinical definite diagnosis.

Given that TSC is a benign disease that does not cause related symptoms, no special treatment was provided to this patient. At a follow-up appointment on April 2021, the patient's condition had not deteriorated further, and did not display any new clinical manifestations. The patient had elected to have laboratory and imaging tests at a local hospital during the follow-up period, but refused to provide these results. All procedures performed in studies involving human participants were in accordance with the ethical standards of the institutional and/or national research committee(s) and with the Helsinki Declaration (as revised in 2013). Written informed consent was obtained from the patient for the publication of this case report and any accompanying images. A copy of the written consent form is available for review by the editorial office of this journal.

\section{Discussion}

TSC is a rare autosomal dominant multisystemic disorder, characterized by a neurocutaneous syndrome, seizures, cognitive disability, and the formation of benign hamartomas lesions in various organs, primarily, the brain, skin, kidneys, heart, lungs, and eyes (1). A diagnostic evaluation may be initiated due to a positive family history or clinical signs. At the 2012 International TSC Consensus Conference, the diagnostic criteria for TSC were updated (3). In this case, the patient had three major features (hypomelanotic macules, ungual fibromas, and SENs), and 1 minor feature (multiple renal cysts). In addition, the osteogenic destruction of the skull and bones, multiple solid nodules in the lungs, and the presence of ungual fibromas in immediate relatives were also suggestive of TSC.

TSC is caused by the inactivation of mutations in the TSC1 and TSC2 tumor suppressor genes on chromosomes 9 and 16, respectively. In normal eukaryotic cells, TSC1 and TSC2 genes encode proteins TSC1 (hamartin) and TSC2 (tuberin), respectively. After the formation of the complex, the two proteins can act on Ras homolog enriched in brain (RHeB), and hydrolyze the active form of $\mathrm{RHeB}$-guanosine triphosphate (RHeB-GTP) into the RHeB-guanosine diphosphate (RHeB-GDP) state, thus inhibiting the mechanistic target of rapamycin (mTOR) complex 1 (mTORC1). mTORC1 mediates major processes, including cellular overgrowth, migration, and neuronal excitability (5). Thus, when TSC1 or TSC2 genes are inactivated-mutated, mTORC1 and the downstream signaling pathway are overactivated, resulting in the occurrence of TSC. The inhibition of mTORC1 with allosteric inhibitors, such as everolimus and sirolimus, is recommended for TSC management $(6,7)$. The overactivation of phosphatidylinositol-3-kinase and protein kinase $\mathrm{B}(\mathrm{PI} 3 \mathrm{~K} / \mathrm{Akt}) /$ the mTOR signaling pathway is prevalent in the occurrence and development of various human tumors.

As an important serine/threonine protein kinase and the downstream signal of PI3K/Akt, mTOR controls the growth, proliferation, apoptosis, invasion, and metastasis of tumor cells by regulating the phosphorylation levels of 
S6 kinase 1 (S6K1), eukaryotic translation initiation factor 4E-binding protein 1 (4EBP1) and other transcriptional regulators (8). Usually, TSC leads to the formation of benign tumors, but malignant tumors with TSC are rarely reported (9-11). Indeed, to date, only a small number of cases have been reported (9-11). Kuroda et al. reported a pediatric case of osteosarcoma and TSC in which a female was diagnosed with TSC at the age of 6 years due to hypomelanotic macules and SENs that later developed to the angiofibromatosis of the face, renal angiomyolipoma, and SEGA (9). The patient was diagnosed with osteosarcoma of the left femur at 12 years of age (9). Gene sequencing revealed a somatic missense mutation in the TP53 gene combined with a novel germline mutation in the TSC2 gene (9).

To our knowledge, this is the first reported case of CC combined with TSC. Based on the patient's 15 -year long history of feet tumors, we speculated that there might be a common mechanism between the pathogenesis and development of TSC and CC. A previous study indicated that human papillomavirus 16 (HPV16) E6 interacts with TSC2 (tuberin) (12). High-risk HPV infection is the necessary condition for CC, and oncoproteins encoded by HPV E6 play a vital role in carcinogenesis. In that study, Lu et al. suggested that E6 protein binds to the tumor suppressor protein, tuberin, and causes the proteasomemediated degradation of tuberin, leading to the activation of S6 kinase downstream of the Akt signaling pathway (12). In addition, Luo et al. reported that TSC1 loss facilitates the p53 haploinsufficiency-mediated activation of the Pten/PI3K/Akt/mTOR axis and its downstream target Abcc4, leading to hepatocellular carcinoma tumorigenesis and metastasis (13). Further studies need to be conducted to better elucidate the association between TSC and malignancy; however, a few studies seem to suggest that alterations in the mTOR pathway and a secondary "hit", such as TP53 mutation HPV infection, may contribute to malignancy formation.

TSC is characterized by the formation of benign tumors, and oncologists should pay attention to its special imaging manifestations to distinguish it from metastasis. Such benign tumors often involve multiple organs, including the skin, brain, kidneys, heart, and lungs (14). In the central nervous system, SENs, SEGA, and cerebral cortex dysplasia are often present, and the former three are often accompanied by calcification. Angiomyolipomas and multiple renal cysts are common in the kidney. In the lung, LAM, multifocal micronodular pneumocyte hyperplasia, and hyalinoma of the lung are the main features. It should be noted that the skull and vertebral body were observed to be involved in the present case (see Figure $3 A, 3 B, 3 E$ ); however, bone involvement is rarely described in TSC. The number of studies related to the imaging findings of bone changes is very limited, and the bone changes in TSC is easy to be ignored or misdiagnosed as bone metastasis.

Boronat et al. reviewed 92 TSC patients, and scleroticbone-lesions (SBLs) were found in 82 cases (89\%), and mainly affected the posterior vertebral elements (15). Brakemeier et al. analyzed the CT data sets of 49 TSC patients and also suggested that SBLs could serve as a potential imaging biomarker for the diagnosis of TSC (16). The SBLs associated with TSC often occur in cancellous bone rich areas, such as the skull, pelvis, and vertebral body. It is speculated that SBL is related to trabecular bone fusion with abnormal plasticity during bone development. In our case, multiple SBLs, with clear boundaries, were observed on the skull and vertebral body, and the patient lacked corresponding pain symptoms. The clear boundaries and lack of pain of bone lesions are consistent with previous studies. In addition, some atypical imaging features were also observed, such as multiple non-calcified nodules in the lungs (see Figure 3C), hepatic angiomyolipoma (multiple heterogeneous nodules with heterogeneous enhancement) (see Figure 3F), and thyroid nodules.

The diagnosis of TSC constitutes a challenge to oncologists because of the rarity and diversity. A detail medical examination and high index of suspicion are required to make the diagnosis. In order to reduce the misdiagnosis of TSC, we suggest oncologists to detect the characteristic features such as hypomelanotic macules, ungual fibromas, SENs and retinal hamartomas. The management of TSC should be multidisciplinary in response to individual symptoms. Several drugs have been used to treat TSC-associated epilepsy, SEGAs, pulmonary LAM, renal angiomyolipomas and dermatological manifestations. These mainly include anticonvulsant medication and mTOR inhibitors (17). Note that treatment of TSC requires a balance of benefits and toxicity, and not all patients need treatment. There are some limitations in our report. Unfortunately, the patient elected to have laboratory and imaging tests at a local hospital during the follow-up period and refused to provide these results.

\section{Conclusions}

We presented a case of CC with TSC, which was 
misdiagnosed as multiple metastases. In this case, toe masses, pelvic nodules, and multiple osteogenic lesions were initially observed. Combined with the patient's CC history, metastasis was preliminarily diagnosed based on imaging and clinical observations. After the pathology results returned no evidence of malignancy, hypomelanotic macules on the back, SENs, ungual fibromas, multiple renal cysts, and SBLs of the skull and vertebrae were observed, which led to the diagnosis of TSC. The patient had no organ function damage and did not have to undergo treatment. After a follow-up period of almost 65 months, the patient's quality of life was good. This article discussed the imaging features of TSC, its molecular mechanisms, and its potential association with CC. Malignancy is not a clinical manifestation of TSC; however, a review of published case reports revealed several reports of malignancy combined with TSC. Oncologists should pay more attention to the differential diagnosis of benign diseases, such as TSC, in the face of multiple lesions and atypical symptoms to reduce misdiagnosis and overtreatment.

\section{Acknowledgments}

Funding: None.

\section{Footnote}

Reporting Checklist: The authors have completed the CARE reporting checklist. Available at https://dx.doi. org/10.21037/apm-21-2814

Conflicts of Interest: All authors have completed the ICMJE uniform disclosure form (available at https://dx.doi. org/10.21037/apm-21-2814). The authors have no conflicts of interest to declare.

Ethical Statement: The authors are accountable for all aspects of the work in ensuring that questions related to the accuracy or integrity of any part of the work are appropriately investigated and resolved. All procedures performed in studies involving human participants were in accordance with the ethical standards of the institutional and/or national research committee(s) and with the Helsinki Declaration (as revised in 2013). Written informed consent was obtained from the patient for the publication of this case report and any accompanying images. A copy of the written consent form is available for review by the editorial office of this journal.
Open Access Statement: This is an Open Access article distributed in accordance with the Creative Commons Attribution-NonCommercial-NoDerivs 4.0 International License (CC BY-NC-ND 4.0), which permits the noncommercial replication and distribution of the article with the strict proviso that no changes or edits are made and the original work is properly cited (including links to both the formal publication through the relevant DOI and the license). See: https://creativecommons.org/licenses/by-nc-nd/4.0/.

\section{References}

1. Henske EP, Jóźwiak S, Kingswood JC, et al. Tuberous sclerosis complex. Nat Rev Dis Primers 2016;2:16035.

2. Wataya-Kaneda M, Tanaka M, Hamasaki T, et al. Trends in the prevalence of tuberous sclerosis complex manifestations: an epidemiological study of 166 Japanese patients. PLoS One 2013;8:e63910.

3. Northrup H, Krueger DA; International Tuberous Sclerosis Complex Consensus Group. Tuberous sclerosis complex diagnostic criteria update: recommendations of the 2012 Iinternational Tuberous Sclerosis Complex Consensus Conference. Pediatr Neurol 2013;49:243-54.

4. Olubunmi OA. Misdiagnosis of tuberous sclerosis in a Nigerian girl: a case report and review of literature. Ann Afr Med 2010;9:95-101.

5. Liu GY, Sabatini DM. mTOR at the nexus of nutrition, growth, ageing and disease. Nat Rev Mol Cell Biol 2020;21:183-203.

6. Bissler JJ, Kingswood JC, Radzikowska E, et al. Everolimus for angiomyolipoma associated with tuberous sclerosis complex or sporadic lymphangioleiomyomatosis (EXIST-2): a multicentre, randomised, double-blind, placebo-controlled trial. Lancet 2013;381:817-24.

7. Dabora SL, Franz DN, Ashwal S, et al. Multicenter phase 2 trial of sirolimus for tuberous sclerosis: kidney angiomyolipomas and other tumors regress and VEGF- D levels decrease. PLoS One 2011;6:e23379.

8. Condon KJ, Sabatini DM. Nutrient regulation of mTORC1 at a glance. J Cell Sci 2019;132:jcs222570.

9. Kuroda K, Moriya K, Nakano T, et al. A pediatric case of osteosarcoma and tuberous sclerosis complex with a novel germline mutation in the TSC2 gene and a somatic mutation in the TP53 gene. Pediatr Blood Cancer 2021;68:e28960.

10. Wu Y, Li H, Yu X, et al. A case of tuberous sclerosis complex with lymphangioleiomyomatosis and renal cell carcinoma. Onco Targets Ther 2020;13:12421-6. 
11. Gonda K, Akama T, Nakamura T, et al. Cluster of differentiation 8 and programmed cell death ligand 1 expression in triple-negative breast cancer combined with autosomal dominant polycystic kidney disease and tuberous sclerosis complex: a case report. J Med Case Rep 2019; 13:381.

12. $\mathrm{Lu} \mathrm{Z,} \mathrm{Hu} \mathrm{X,} \mathrm{Li} \mathrm{Y,} \mathrm{et} \mathrm{al.} \mathrm{Human} \mathrm{papillomavirus} 16$ E6 oncoprotein interferences with insulin signaling pathway by binding to tuberin. J Biol Chem 2004;279:35664-70.

13. Luo YD, Fang L, Yu HQ, et al. p53 haploinsufficiency and increased mTOR signalling define a subset of aggressive hepatocellular carcinoma. J Hepatol 2021;74:96-108.

14. Krueger DA, Northrup H; International Tuberous Sclerosis Complex Consensus Group. Tuberous sclerosis complex surveillance and management: recommendations of the 2012 International Tuberous Sclerosis Complex
Consensus Conference. Pediatr Neurol 2013;49:255-65.

15. Boronat S, Barber I, Thiele EA. Sclerotic bone lesions in tuberous sclerosis complex: A genotype-phenotype study. Am J Med Genet A 2017;173:1891-5.

16. Brakemeier S, Vogt L, Adams LC, et al. Sclerotic bone lesions as a potential imaging biomarker for the diagnosis of tuberous sclerosis complex. Sci Rep 2018;8:953.

17. Kingswood JC, Jozwiak S, Belousova ED, et al. The effect of everolimus on renal angiomyolipoma in patients with tuberous sclerosis complex being treated for subependymal giant cell astrocytoma: subgroup results from the randomized, placebo-controlled, Phase 3 trial EXIST-1. Nephrol Dial Transplant 2014;29:1203-10.

(English Language Editor: L. Huleatt)
Cite this article as: Ye T, Feng L, Peng M, Yuan S, Chen Y, Liu X, Chen J. Tuberous sclerosis complex misdiagnosed as multiple metastases in a cervical cancer patient: case report and literature review. Ann Palliat Med 2021;10(10):11232-11238. doi: 10.21037/apm-21-2814 